\title{
Association between expression of nuclear receptor co-activator 5 protein and prognosis in postoperative patients with osteosarcoma
}

\author{
YA WU ${ }^{1,2^{*}}$, JIAN WU $^{3^{*}}$, QI-RONG DONG ${ }^{1}$ and NAI-ZHOU GUO ${ }^{3}$ \\ ${ }^{1}$ Department of Orthopedics, Second Affiliated Hospital of Suzhou University, Suzhou, Jiangsu 215004; Departments of \\ ${ }^{2}$ Orthopedics and ${ }^{3}$ Laboratory Medicine, The First People's Hospital of Yancheng City, Yancheng, Jiangsu 224005, P.R. China
}

Received June 2, 2016; Accepted August 1, 2017

DOI: 10.3892/ol.2017.7513

\begin{abstract}
The aim of the present study was to investigate the association between the expression of nuclear receptor co-activator 5 protein (NCOA5) and the prognosis of postoperative patients with osteosarcoma. Human osteosarcoma samples were collected from 145 patients and normal bone tissues were collected from 100 individuals as controls. Immunohistochemistry (IHC) and reverse transcription-polymerase chain reaction (RT-PCR) were employed to measure the levels of NCOA5 protein in cases of human osteosarcoma. The results from the RT-PCR analysis demonstrated that the positive rate of NCOA5 mRNA expression in human osteosarcoma was $17.24 \%(25 / 145)$. The positive rate in normal bone tissues was $84.00 \%$ (84/100), which was significantly higher compared with that of human osteosarcoma tissues $\left(\chi^{2}=33.166\right.$; $\mathrm{P}<0.001)$. IHC staining indicated that the positive rate of NCOA5 protein in the osteosarcoma samples was $26.21 \%$ (38/145). The positive rate in normal bone tissues was $82.00 \%$ $(82 / 100)$, which was significantly increased compared with that of human osteosarcoma tissues $\left(\chi^{2}=28.166 ; \mathrm{P}<0.001\right)$. NCOA5 mRNA and protein expression levels were consistent in human osteosarcoma tissues, and were lower than in control tissues. The expression of NCOA5 was low in human osteosarcoma tissues, while it was high in normal bone tissues. These low NCOA5 expression levels were associated with postoperative survival of human osteosarcoma.
\end{abstract}

Correspondence to: Dr Qi-Rong Dong, Department of Orthopedics, Second Affiliated Hospital of Suzhou University, 1055 Sanxiang Road, Suzhou, Jiangsu 215004, P.R. China

E-mail: 65141271@qq.com

Dr Nai-Zhou Guo, Department of Laboratory Medicine, The First People's Hospital of Yancheng City, 166 Yulong Road, Yancheng, Jiangsu 224005, P.R. China

E-mail: piaoxue1982717@sina.com

${ }^{*}$ Contributed equally

Key words: human osteosarcoma, nuclear receptor co-activator 5, prognosis, immunohistochemistry

\section{Introduction}

Osteosarcoma is the most common primary tumor of bone tissue; it is most common in young people and exhibits a high degree of malignancy $(1,2)$. The treatment of osteosarcoma is neoadjuvant chemotherapy and surgery (3). The five-year survival rate remains at 60-70\%, and there has been no significant increase during the past 10 years (4). The primary reason for this is that the mechanism of pathogenesis is not clear. The formation and development of the tumor is a multifactorial, multi-stage and a gradual process; early diagnosis and timely intervention are essential for the prognosis of patients (5). In previous years, the associations between cell proliferation and cell signaling have become an area of interest $(6,7)$.

Previous studies have identified that nuclear receptor co-activator 5 (NCOA5) is a nuclear receptor co-regulator that is widely expressed $(8,9)$. NCOA5 is also a co-regulator for the estrogen receptor and orphan nuclear hormone receptor co-activator BD73 (10). The transcription of NCOA5 is mediated by estrogen receptors to achieve homeostatic regulation of human B cells. NCOA5 gene defects may lead to cancer and diabetes $(11,12)$.

To investigate the association between the expression of NCOA5 and the prognosis of postoperative patients with osteosarcoma, immunohistochemistry (IHC) and reverse transcription-polymerase chain reaction (RT-PCR) were employed in the present studyt to measure the levels of NCOA5 protein in patients with human osteosarcoma, using normal bone tissues as the controls. The association between these groups and the mechanism of the formation of osteosarcoma were explored to create novel targets for early diagnosis of this disease.

\section{Patients and methods}

Patient samples. Samples of human osteosarcoma were collected between February 2012 and June 2017 from 145 patients who received surgical resection at The First People's Hospital of Yancheng City (Yancheng, China), and had been diagnosed by pathological confirmation. Detailed clinical data were available for all the patients, including sex, age, differentiation, invasion depth, lymph node metastasis and Union for International Cancer Control stage tumor node metastasis (TNM) stage (13), and none had received preoperative chemotherapy or radiotherapy. Human osteosarcoma patients 
included 75 males and 70 females, (age range between 15-63 years; average age, $40.9 \pm 11.6$ years). Normal bone tissue specimens were collected by surgical resection from 100 individuals to serve as a control group. These included 55 males and 45 females, (age range 16-68 years; average age, $34.4 \pm 10.3$ years). No statistically significant differences were detected in age or sex between the two groups. The protocol used in the present study was in accordance with the Medical Ethics and Human Clinical Trial Committee of The First People's Hospital of Yancheng City, (Identification No. HMU Ethics 20120003). Written informed consent was obtained by all participants in the present study.

Immunohistochemical staining. The EnVision and DAB chromogenic reagent kit (Agilent Technologies, Inc., Santa Clara, CA, USA) was used for immunostaining to detect the distribution of NCOA5 (Antibody Diagnostic; Applied Biosystems; Thermo Fisher Scientific, Inc., Waltham, MA, USA). Immunohistochemical procedures were performed in strict accordance with the manufacturer's protocol (Applied Biosystems; Thermo Fisher Scientific, Inc.). The EnVision and 3,3'-diaminobenzidine chromogenic reagent kits (Antibody Diagnostic; Applied Biosystems) were used for immunohistochemical staining. All slide staining was performed under the same conditions: The tissue was sliced to $4-\mu \mathrm{m}$ thickness, dehydrated with $21 \%$ sucrose, de-waxed and subjected to antigen retrieval in $0.01 \mathrm{~mol} / 1 \mathrm{citric}$ acid $\mathrm{pH} 6.0$ at $95^{\circ} \mathrm{C}$ for $10 \mathrm{~min}$. Normal goat serum (10\%; Toyobo Life Science, Osaka, Japan) was placed on the slide and incubated for $10 \mathrm{~min}$ at room temperature. Slides were then incubated with the corresponding specific primary antibodies (mouse anti-osteonection/NCOA5; cat. no. ab70831; Abcam, Cambridge, UK; 1:1,000) for $1.5 \mathrm{~h}$ at room temperature. Tissue sections were then washed with PBS 3 times for $3 \mathrm{~min}$. Subsequently, tissue sections were incubated with secondary antibody horseradish peroxidase-conjugated mouse anti-osteonection/NCOA5 IgG (1:1,000; cat. no. ab125508; Abcam) for $30 \mathrm{~min}$ at room temperature. Proteins were detected using 3,3'-Diaminobenzidine (5 min at room temperature), while the nucleus was stained with $10 \%$ hematoxylin at $37^{\circ} \mathrm{C}$ for $7 \mathrm{~min}$. Tissue samples were then dehydrated in $75,85,95$ and $100 \%$ gradient ethanol series, cleared by $1 \%$ xylene and sealed with rubber cement. Each batch of staining contained a positive control sourced from patients with confirmed osteosarcoma [with the known positive section reagent (cat. no. ab92431; Abcam; 1:1,000)] and a negative control, in which the corresponding specific antibody was replaced by PBS.

Samples with nuclei with yellow or tan reactant particles were considered positive. A total of four independent experiments were conducted, and four fields of view were assessed by optical microscopy at x200 magnification. Analysis of the staining scores was semi-quantitative: Final staining scores were calculated by multiplying the positive cell percentage score and the staining intensity score. The positive cell staining percentage score criteria were as follows: $0-15 \%, 0$; $>15-30 \%, 1 ;>30-45 \%, 2$; and $>45 \%, 3$. These staining scores were then classified as follows: $>2$, negative $(-) ; 2-4$, weakly positive $(+)$; 4-6, positive $(++)$; and $\geq 6$, strongly positive $(+++)$. For the convenience of statistical analysis, samples within the
(-) group were defined as the negative expression group (-), and samples within the $(+),(++)$ and $(+++)$ groups were defined as the positive expression group $(+)$.

Detection of NCOA5 mRNA expression by RT-PCR. Total RNA was isolated from tissues using TRIzol ${ }^{\circledR}$ reagent (NanoDrop Technologies; Thermo Fisher Scientific, Inc.) according to the manufacturer's protocol and was quantified using a NanoDrop spectrophotometer (NanoDrop Technologies; Thermo Fisher Scientific, Inc.). A total of $2 \mu \mathrm{g}$ RNA was reverse transcribed to complementary DNA using the QuantiNova Reverse Transcription kit (50) according to the manufacturer's protocol (cat. no. 205411; Qiagen GmbH, Hilden, Germany). Samples were then amplified by semi-quantitative PCR with $\beta$-actin as a reference. The sequences of the primers (Sangon Biotech., Co., Ltd., Shanghai, China) are listed in Table I. Thermocycler conditions were as follows: Pre-denaturation at $94^{\circ} \mathrm{C}$ for $4 \mathrm{~min}$, followed by 30 cycles of $94^{\circ} \mathrm{C}$ for $10 \mathrm{sec}, 55^{\circ} \mathrm{C}$ for $30 \mathrm{sec}$ and $72^{\circ} \mathrm{C}$ for $60 \mathrm{sec}$.

Amplification of NCOA5 by PCR was examined using $1 \%$ agarose gel electrophoresis. The absorbance value of the sample gene and the reference ( $\beta$-actin) were read, and the results were expressed as the ratio (sample value/reference value). If the ratio of osteosarcoma to reference absorbance values was positive, NCOA5 mRNA expression was considered to be positive; if it was negative, NCOA5 mRNA expression of the sample was categorized as negative.

Statistical methods. SPSS 13.0 statistical software (SPSS, Inc., Chicago, IL, USA) was used for statistical analysis. The $\chi^{2}$ test was used to compare the distribution of NCOA5 between osteosarcoma and normal bone tissues. The Kaplan-Meier survival analysis with log-rank test was performed to analyze the association between the expression levels of the proteins in cancer tissue or other clinicopathological characteristics and the survival rate of patients. Associations between the expression of NCOA5 and overall survival of postoperative patients with osteosarcoma were assessed using Cox regression analysis. $\mathrm{P}<0.05$ was considered to indicate a statistically significantly difference.

\section{Results}

Cell nucleus staining distribution of NCOA5 in human osteosarcoma and normal bone tissues. The positive rate of NCOA5 staining in human osteosarcoma tissues was $26.21 \%$ (38/145), which was significantly lower compared with the positive rate of NCOA5 in normal bone tissues, which was $82.00 \%$ (82/100; $\mathrm{P}<0.05$; Fig. 1).

NCOA5 mRNA expression in human osteosarcoma and normal bone tissues. The results from RT-PCR indicate that mRNA NCOA5 was expressed in osteosarcoma and normal bone tissues $(\mathrm{P}<0.05$; Table I). The positive rate of NCOA5 mRNA expression in osteosarcoma was $17.24 \%(25 / 145)$, which was significantly lower compared with that in normal bone tissue (84.00\%, 84/100; $\chi^{2}=33.166$; $\mathrm{P}<0.001$; Fig. 2). Compared with the normal bone tissue, the rate of NCOA5 expression was significantly lower in pulmonary metastatic osteosarcoma tissues. 
Table I. Primer sequences for reverse transcription-polymerase chain reaction analysis.

\begin{tabular}{llc}
\hline Primers & Primer sequences (5'-3') & $\begin{array}{c}\text { Product } \\
\text { size (bp) }\end{array}$ \\
\hline NCOA5 & & \\
Forward & CTGCTGGCAGACAACAGGTA & 344 \\
Reverse & CTGTTTGCTGCTGTGGAAAA & 344 \\
B-actin & & \\
Forward & TGACGTGGACATCCGCAAAG & 231 \\
Reverse & CTGGAAGGTGGACAGCGAGG & 231 \\
\hline
\end{tabular}

NCOA5, nuclear receptorco-activator 5 .
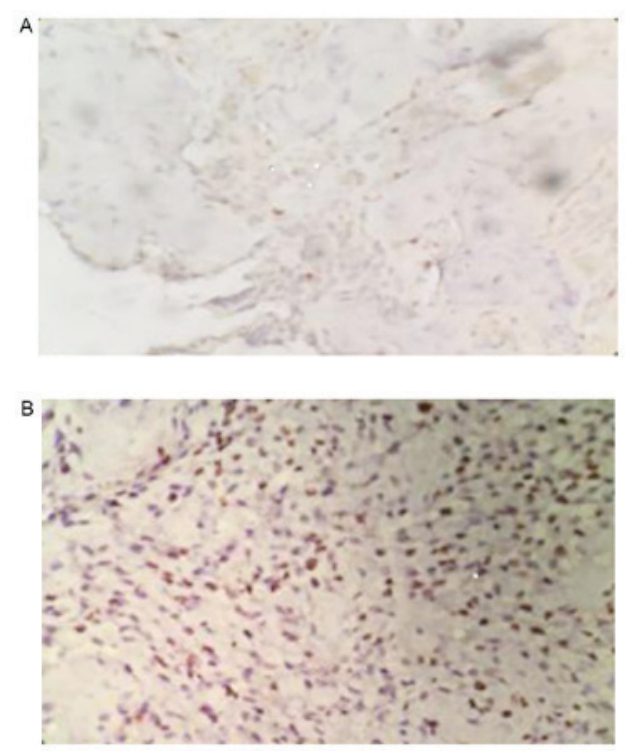

Figure 1. EnVision immunohistochemistry of NCOA5 staining in human osteosarcoma and normal bone tissues at x200 magnification. (A) Weak positive staining result for NCOA5 in human osteosarcoma. (B) Strong positive staining result for NCOA5 in normal bone tissues. NCOA5, nuclear receptor co-activator 5 .

Association between the expression of NCOA5 $m R N A$ and the clinicopathological characteristics of osteosarcoma. The expression levels of NCOA5 mRNA and protein in osteosarcoma were consistent and were lower than those in the normal controls. NCOA5 exhibited a low level of expression in osteosarcoma, and was not associated with sex, age or tumor size. However, it was associated with recurrence and metastasis $(\mathrm{P}<0.01$; Table II).

Association between the expression of NCOA5 and overall survival of postoperative patients with osteosarcoma. The overall survival time of NCOA5-positive patients was $(60.92 \pm 3.45)$ months after a median follow-up of 61.5 months $(61.5 \pm 77.3$ months). The overall survival of NCOA5-negative patients was $(25.68 \pm 5.65)$ months. Kaplan-Meier survival analysis demonstrated that there was a significant difference between these data $\left(\chi^{2}=0.990\right.$; $\mathrm{P}=0.031$; Fig. 3).
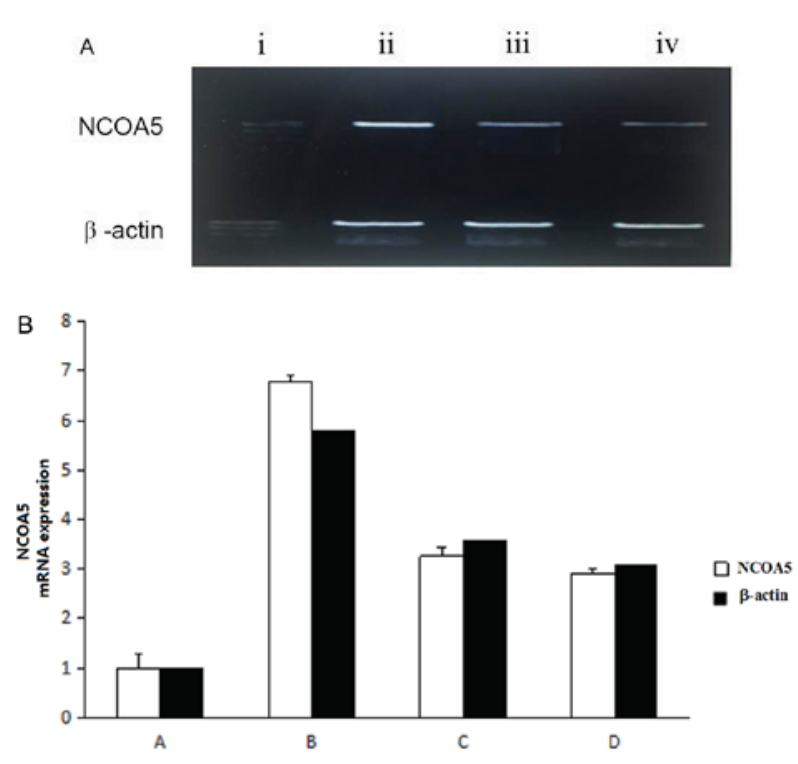

Figure 2. NCOA5 mRNA expression in osteosarcoma and normal bone tissues. (A) Western blot analysis and (B) quantification of mRNA expression of the (i) negative control group, (ii) normal bone tissue group, (iii) human osteosarcoma without pulmonary metastasis group and (iv) human osteosarcoma with pulmonary metastasis group. NCOA5, nuclear receptor co-activator 5 .

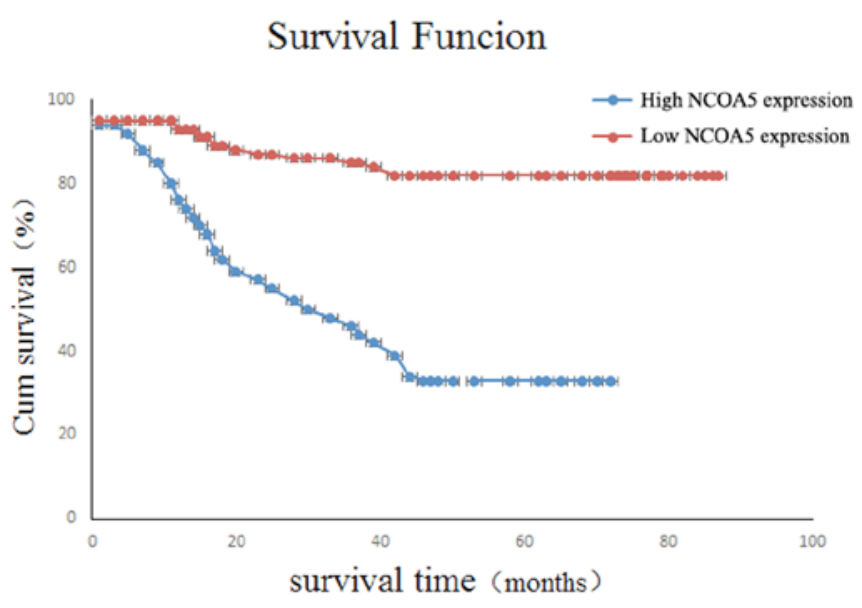

Figure 3. NCOA5 expression and survival analysis of patients with osteosarcoma. NCOA5, nuclear receptor co-activator 5.

In addition, adjuvant therapy, sex, age, gross morphology, tumor invasion depth and lymph node metastasis were not identified as independent factors affecting the overall survival of postoperative patients (Table III).

\section{Discussion}

Previous studies have indicated that the occurrence and development of tumors is a complicated process (14); disorders in the regulation of cell growth and proliferation may affect the occurrence and development of tumors (15). Concurrently, the abnormal expression of tumor-associated genes, and the abnormal activation of cell signal transduction and cell proliferation cycle are also involved in numerous aspects of tumor development $(16,17)$. Cell growth and proliferation in the human body are affected and controlled by a number of 
Table II. Association between NCOA5 mRNA and protein expression with clinicopathological features in osteosarcoma.

\begin{tabular}{|c|c|c|c|c|c|c|c|}
\hline Characteristics & $\mathrm{n}$ & $\begin{array}{c}\text { NCOA5 mRNA } \\
\text { positive rate, } \mathrm{n}(\%)\end{array}$ & $\chi^{2}$ & P-value & $\begin{array}{l}\text { NCOA5 protein } \\
\text { positive rate, } \mathrm{n}(\%)\end{array}$ & $\chi^{2}$ & P-value \\
\hline Sex & & & 0.190 & 0.663 & & 0.008 & 0.927 \\
\hline Male & 75 & $15(20.0)$ & & & $12(16.0)$ & & \\
\hline Female & 70 & $11(15.7)$ & & & $11(15.7)$ & & \\
\hline Age (years) & & & 0.121 & 0.728 & & 0.005 & 0.945 \\
\hline$<40$ & 73 & $13(17.8)$ & & & $12(16.4)$ & & \\
\hline$\geq 40$ & 72 & $10(13.9)$ & & & $13(18.1)$ & & \\
\hline Tumor diameter $(\mathrm{cm})$ & & & 0.351 & 0.553 & & 0.072 & 0.789 \\
\hline$\geq 10$ & 63 & $11(17.5)$ & & & $10(15.9)$ & & \\
\hline$<10$ & 82 & $13(15.9)$ & & & $15(18.3)$ & & \\
\hline Pulmonary metastasis & & & 7.601 & 0.006 & & 7.601 & 0.006 \\
\hline Yes & 65 & $26(40.0)$ & & & $26(40.0)$ & & \\
\hline No & 80 & $5(6.3)$ & & & $5(6.3)$ & & \\
\hline
\end{tabular}

The $\chi^{2}$ test was used to compare the distribution of NCOA5 with clinicopathological features in osteosarcoma. P<0.05 was considered to indicate a statistically significant difference. NCOA5, nuclear receptor co-activator 5.

Table III. Analysis of survival factors in patients with osteosarcoma.

\begin{tabular}{|c|c|c|c|c|c|c|c|c|}
\hline \multirow[b]{2}{*}{ Clinicopathological features } & \multicolumn{4}{|c|}{ Univariate analysis } & \multicolumn{4}{|c|}{ Multivariate analysis } \\
\hline & B & SE & P-value & $95 \% \mathrm{CI}$ & B & SE & $\mathrm{P}$-value & $95 \% \mathrm{CI}$ \\
\hline Age & 0.658 & 0.604 & 0.269 & & 0.612 & 0.598 & 0.325 & \\
\hline Sex & 0.060 & 0.602 & 0.940 & & 0.068 & 0.615 & 0.915 & \\
\hline Tumor size & 1.756 & 0.765 & 0.042 & $1.02-3.08$ & 1.211 & 0.544 & 0.031 & $1.06-3.13$ \\
\hline Tumor location & 0.052 & 0.512 & 0.819 & & 0.063 & 0.610 & 0.875 & \\
\hline Pathological type & 0.598 & 0.512 & 0.294 & & 0.626 & 0.651 & 0.312 & \\
\hline Lymph node metastasis & 1.796 & 0.621 & $<0.001$ & $1.42-4.26$ & 1.826 & 0.631 & $<0.001$ & $1.55-4.52$ \\
\hline Low NCOA5 expression & 3.003 & 1.071 & 0.003 & $1.41-6.08$ & 3.012 & 1.076 & 0.003 & $1.48-6.23$ \\
\hline
\end{tabular}

The Kaplan-Meier survival analysis with log-rank test was used to perform univariate analysis examining the association between the expression levels of the proteins in cancer tissue or other clinicopathological characteristics and the survival rate of patients. $\mathrm{P}<0.05$ was considered to indicate a statistically significant difference. CI, confidence interval; NCOA5, nuclear receptor co-activator 5; SE, standard error; B, regression coefficient.

factors $(18,19)$. In particular, cell signaling proteins, growth factors and their receptors, apoptotic proteins and transcription factors, and the changes to these factors are closely associated with the occurrence and development of tumors $(20,21)$.

Previous studies have also revealed that the NCOA5 protein may be associated with interleukin (IL)-6, tumor necrosis factor (TNF)- $\alpha$ and nuclear factor (NF)- $\mathrm{B}$ (22-24). Prevention of the overexpression of IL- 6 may limit the occurrence and development of certain types of cancer. TNF- $\alpha$ was significantly increased in NCOA5 gene-deficient animal tumors. However, this gene defect is not reversible in vivo, and the specific mechanism requires additional study (25).

Previous studies have also identified that the decrease in NCOA5 expression in esophageal squamous cell carcinoma (ESCC) tissue is associated with the differentiation status of the tumor and the TNM stage, while is not associated with age, sex, weight loss, tumor location or lymph node metastasis $(26,27)$. In addition, the expression of NCOA5 in normal tissues was higher compared with that in tumor tissues. As the level of tumor differentiation and TNM stage are important indicators of the malignant degree of tumors, the results of the present study suggest that the decreased expression of NCOA5 may be involved in the promotion of tumor progression (28-30).

One limitation of the current study is the relatively small sample size. Nevertheless, to the best of our knowledge, the present study is among the largest studies addressing NCOA5 protein expression in osteosarcoma. The results of the present study indicated that the expression of NCOA5 in osteosarcoma was significantly lower compared with that in normal bone tissue. In addition, the expression levels of NCOA5 protein 
in benign bone tumor tissues were significantly higher than in osteosarcoma tissues, which may indicate an association between the occurrence and development of tumors and low NCOA5 expression.

The present study demonstrated that the expression of NCOA5 protein in human specimens is closely associated with the occurrence of osteosarcoma. The expression of NCOA5 in osteosarcoma tissues was significantly lower compared with that in normal bone tissue. This indicates that NCOA5 may be a tumor-suppressor gene in humans.

The results of the present study suggested that the expression of NCOA5 is consistent in different types of osteosarcoma tissues, such as those with or without pulmonary metastasis. The expression of NCOA5 in osteosarcoma was significantly lower compared to that in normal bone tissue. The low expression of NCOA5 may be a cause of osteosarcoma, and therefore, it may be important to detect the expression of NCOA5 in osteosarcoma for the diagnosis of this disease.

In the present study, it was also identified that the expression of NCOA5 protein in patients with osteosarcoma was associated with survival prognosis, and the clinical features of the tumor were significantly associated with the survival rate, differentiation and staging.

In conclusion, the results of the present study indicate the potential role of NCOA5 in the progression of osteosarcoma, highlighting its low expression as an independent prognostic factor. Furthermore, additional studies examining NCOA5 may also assist in developing novel therapeutic strategies for osteosarcoma.

\section{Acknowledgements}

The present study was supported by Jiangsu Pharmaceutical Association (grant no. 201542) and the Science and Technology commission of Yancheng City (grant no. YK2015002).

\section{References}

1. Huang J, Liu K, Song D, Ding M, Wang J, Jin Q and Ni J: Krüppel-like factor 4 promotes high-mobility group box 1-induced chemotherapy resistance in osteosarcoma cells. Cancer Sci 107: 242-249, 2016.

2. Li YS, Deng ZH, Zeng C and Lei GH: JNK pathway in osteosarcoma: Pathogenesis and therapeutics. J Recept Signal Transduct Res 36: 465-470, 2016.

3. Lamplot JD, Denduluri S, Qin J, Li R, Liu X, Zhang H, Chen X, Wang N, Pratt A, Shui W, et al: The current and future therapies for human osteosarcoma. Curr Cancer Ther Rev 9 : 55-77, 2013.

4. Chakravarthi PS, Kattimani VS, Prasad LK and Satish PR: Juxtacortical osteosarcoma of the mandible: Challenges in diagnosis and management. Natl J Maxillofac Surg 6: 127-131, 2015.

5. Commandeur AE, Styer AK and Teixeira JM: Epidemiological and genetic clues for molecular mechanisms involved in uterine leiomyoma development and growth. Hum Reprod Update 21: 593-615, 2015.

6. Zhou W, Zhu Y, Chen S, Xu R and Wang K: Fibroblast growth factor receptor 1 promotes MG63 cell proliferation and is associated with increased expression of cyclin-dependent kinase 1 in osteosarcoma. Mol Med Rep 13: 713-719, 2016.

7. Yang J, Cheng D, Zhou S, Zhu B, Hu T and Yang Q: Overexpression of X-box binding protein 1 (XBP1 correlates to poor prognosis and up-regulation of PI3K/mTOR in human osteosarcoma. Int J Mol Sci 16: 28635-28646, 2015.
8. Liu CY and Feng GS: NCOA5, a molecular link between type 2 diabetes and liver cancer. Hepatobiliary Surg Nutr 3: 106-108, 2014.

9. Tetel MJ, Auger AP and Charlier TD: Who's in charge? Nuclear receptor coactivator and corepressor function in brain and behavior. Front Neuroendocrinol 30: 328-342, 2009.

10. Gao S, Li A, Liu F, Chen F, Williams M, Zhang C, Kelley Z, Wu CL, Luo R and Xiao H: NCOA5 Haploinsufficiency results in glucose intolerance and subsequent hepatocellular carcinoma. Cancer Cell 24: 725-737, 2013.

11. Lahusen T, Henke RT, Kagan BL, Wellstein A and Riegel AT: The role and regulation of the nuclear receptor co-activator AIB1 in breast cancer. Breast Cancer Res Treat 116: 225-237, 2009.

12. Chauhan C, Zraly CB, Parilla M, Diaz MO and Dingwall AK: Histone recognition and nuclear receptor co-activator functions of Drosophila cara mitad, a homolog of the N-terminal portion of mammalian MLL2 and MLL3. Development 139: 1997-2008, 2012.

13. Chen VW, Ruiz BA, Hsieh MC, Wu XC, Ries LA and Lewis DR: Analysis of stage and clinical/prognostic factors for lung cancer from SEER registries: AJCC staging and collaborative stage data collection system. Cancer 120 (Suppl 23): S3781-S3792, 2014.

14. Chinenov Y, Gupte R and Rogatsky I: Nuclear receptors in inflammation control: Repression by GR and beyond. Mol Cell Endocrinol 380: 55-64, 2013.

15. Pavlin MR, Brunzelle JS and Fernandez EJ: Agonist ligands mediate the transcriptional response of nuclear receptor heterodimers through distinct stoichiometric assemblies with coactivators. J Biol Chem 289: 24771-24778, 2014.

16. Gyamfi MA and Wan YJ: Pathogenesis of alcoholic liver disease: The role of nuclear receptors. Exp Biol Med (Maywood) 235: 547-560, 2010.

17. Brand TM, Iida M, Li C and Wheeler DL: The nuclear epidermal growth factor receptor signaling network and its role in cancer. Discov Med 12: 419-432, 2011.

18. Poos K, Smida J, Maugg D, Eckstein G, Baumhoer D, Nathrath M and Korsching E: Genomic heterogeneity of osteosarcoma-shift from single candidates to functional modules. PLoS One 10: e0123082, 2015.

19. Basu-Roy U, Basilico $C$ and Mansukhani A: Perspectives on cancer stem cells in osteosarcoma. Cancer Lett 338: 158-167, 2013.

20. Zhao R, Ni D, Tian Y, Ni B and Wang A: Aberrant ADAM10 expression correlates with osteosarcoma progression. Eur J Med Res 19: 9, 2014.

21. Talmadge JE and Gabrilovich DI: History of myeloid-derived suppressor cells. Nat Rev Cancer 13: 739-752, 2013.

22. Dhar D, Seki E and Karin M: NCOA5, IL-6, Type-2 diabetes and HCC: The deadly quartet. Cell Metab 19: 6-7, 2014.

23. Cumsille P, Coronel A, Conca C, Quiñinao C and Escudero C: Proposal of a hybrid approach for tumor progression and tumor-induced angiogenesis. Theor Biol Med Model 12: 13, 2015.

24. Arvelo F, Sojo F and Cotte C: Tumour progression and metastasis. Ecancermedicalscience 10: 617, 2016.

25. Gillespie MA, Gold ES, Ramsey SA, Podolsky I, Aderem A and Ranish JA: An LXR-NCOA5 gene regulatory complex directs inflammatory crosstalk-dependent repression of macrophage cholesterol efflux. EMBO J 34: 1244-1258, 2015.

26. Lee E, Pandey NB and Popel AS: Crosstalk between cancer cells and blood endothelial and lymphatic endothelial cells in tumour and organ microenvironment. Expert Rev Mol Med 17: e3, 2015.

27. Marcu LG and Harriss-Phillips WM: In Silico modelling of treatment-induced tumour cell kill: Developments and advances. Comput Math Methods Med 2012: 960256, 2012.

28. Fang M, Yuan J, Peng C and Li Y: Collagen as a double-edged sword in tumor progression. Tumour Biol 35: 2871-2882, 2014.

29. Lin G, Sun XJ, Han QB, Wang Z, Xu YP, Gu JL, Wu W, Zhang GU, Hu JL, Sun WY and Mao WM: Epidermal growth factor receptor protein overexpression and gene amplification are associated with aggressive biological behaviors of esophageal squamous cell carcinoma. Oncol Lett 10: 901-906, 2015.

30. Shiomi H, Eguchi Y, Tani T, Kodama M and Hattori T: Cellular distribution and clinical value of urokinase-type plasminogen activator, its receptor, and plasminogen activator inhibitor- 2 in esophageal squamous cell carcinoma. Am J Pathol 156: 567-575, 2000 . 